\title{
On the statistical analysis of Feigenbaum constants
}

\author{
Fatin Sezgin ${ }^{\mathrm{a}}$, Tevfik Metin Sezgin ${ }^{\mathrm{b}, *}$ \\ ${ }^{a}$ School of Applied Technology and Management, Bilkent University, Ankara, Turkey \\ ${ }^{\mathrm{b}}$ CSAIL, Massachusetts Institute of Technology, Cambridge, MA 02139, USA
}

Received 3 November 2005; accepted 11 October 2006

\begin{abstract}
We present statistical analysis of blocks in the binary expansions of Feigenbaum constants $\alpha$ and $\delta$ for the logistic map. The analysis is carried out on both 1016 and 3400 bit expansions. A $\chi^{2}$ test is applied for lumping data and a serial test is applied on gliding data. Contrary to a previous research by Karamanos and Kotsireas, our test results did not indicate any evidence to reject randomness of these constants. Additional 25 randomness tests also support the conjecture of randomness of these constants having transcendental character.
\end{abstract}

(C) 2006 The Franklin Institute. Published by Elsevier Ltd. All rights reserved.

PACS: $02.50 .-\mathrm{r}, 02.50 . \mathrm{Ng}, 05.10 .-\mathrm{a}$

Keywords: Feigenbaum constants; Normal numbers; Random number generation; Statistical analysis

In a recent article Karamanos and Kotsireas [1] studied statistical properties of Feigenbaum constants and they concluded that the available digits of $\alpha$ and $\delta$ show nonrandom behavior.

There are two serious issues to be pointed out in the work of Karamanos and Kotsireas.

(1) They refer to 1016 decimal digit expansions obtained by Broadhurst [2]. Based on our personal communication with Broadhurst, this is the best available estimate of Feigenbaum constants at present. But Karamanos and Kotsireas did not use the full information in this series. Since they took only 1016 binary digits, the obtained analysis corresponds to only about $1 / 3$ of the decimal information. In order to attain equivalent

DOI of original article: $10.1016 / \mathrm{j}$.jfranklin.2006.06.002

*Corresponding author. Tel.: + 16172532663 .

E-mail address: mtsezgin@csail.mit.edu (T. Metin Sezgin). 
information, binary expansion should be extended to at least $1016 \times \log (10) /$ $\log (2)=3382$ binary digits.

(2) The inferences from the statistical analyses are incorrect. Tables presented by the authors do not support their conclusions. For example in the first table the calculated $\chi^{2}$ value is 1.421 as stated. But this value fails to reject the hypothesis of equidistribution. The degrees of freedom is one (not 2 as stated by authors), and a rejection will be possible only for $\chi^{2}>3.841$ with a 5\% Type-I error. Therefore for the rejection of equidistribution, more extreme values are needed. In particular, the table gliding value of block 0 should be larger than 0.531 and the value of block 1 should be less than 0.469. Moreover, there is a missing line in the second table of $\alpha$, after the block 010 we must insert 011 having lumping value 0.136 and gliding value 0.124 . In summary, we get the following test statistics for lumping data of Feigenbaum constants:

\begin{tabular}{llllrl}
\hline & $\begin{array}{l}\text { Block } \\
\text { size }\end{array}$ & $\begin{array}{l}\text { Calculated } \\
\chi^{2}\end{array}$ & $\begin{array}{l}\text { Degrees of } \\
\text { freedom }\end{array}$ & Table $\chi^{2}$ & Result \\
\hline Analysis for $\alpha$ & 1 & 1.421 & 1 & 3.841 & Not significant \\
& 2 & 1.433 & 3 & 7.815 & Significant $(5 \%)$ \\
& 3 & 5.858 & 7 & 14.067 & Not significant \\
Analysis for $\delta$ & 4 & 8.855 & 15 & 24.996 & Not significant \\
& 1 & 0.567 & 1 & 3.841 & Not significant \\
& 2 & 0.740 & 3 & 7.815 & Not significant \\
& 3 & 6.852 & 7 & 14.067 & Not significant \\
& 4 & 8.803 & 15 & 24.996 & Not significant \\
\hline
\end{tabular}

According to our conclusion, contrary to the authors' inference, the present data do not give any evidence to reject the hypothesis of randomness for the digits of Feigenbaum constants.

We carried out calculations and explored the expansion up to 3400 binary digits. In this case also our inferences remain valid. The $\chi^{2}$ values obtained are as follows:

\begin{tabular}{llrlrl}
\hline & Block size & $\begin{array}{l}\text { Calculated } \\
\chi^{2}\end{array}$ & $\begin{array}{l}\text { Degrees of } \\
\text { freedom }\end{array}$ & Table $\chi^{2}$ & Result \\
\hline Analysis for $\alpha$ & 1 & 3.060 & 1 & 3.841 & Not significant \\
& 2 & 8.682 & 3 & 7.815 & Significant $(5 \%)$ \\
Analysis for $\delta$ & 1 & 9.658 & 7 & 14.067 & Not significant \\
& 4 & 17.275 & 15 & 24.996 & Not significant \\
& 2 & 0.142 & 1 & 3.841 & Not significant \\
& 3 & 0.692 & 3 & 7.815 & Not significant \\
& 4 & 7.065 & 7 & 14.067 & Not significant \\
& 9.934 & 15 & 24.996 & Not significant \\
\hline
\end{tabular}


Therefore except for one case, all test statistics fail to reject the randomness hypothesis. Since the hypothesis testing used here has a 5\% level of Type-I error, we must consider some occasional rejections as natural. Because when the number of tests increases the probability of rejecting a true null hypothesis will increase as well. Since we conducted 8 statistical tests, each having a probability 0.05 of rejecting a true null hypothesis, under the assumption of independence between tests, the overall Type-I error will be $1-0.95^{8}=0.34$ approximately, which is a rather large value.

The analysis of gliding data can be accomplished by using the Serial Test proposed by Good [3]. We carried out this analysis too and observed that the two $\chi^{2}$ statistics obtained for the full series of 3400 bits are 8.005 (with $8 \mathrm{df}$ ) and 1.118 (with $4 \mathrm{df}$ ) for the constant $\alpha$. The corresponding statistics for the constant $\beta$ are 6.880 and 0.563 with the same degrees of freedoms. These facts support our assertions on the randomness of Feigenbaum constants. Apart from these we conducted 25 additional empirical tests used in statistical evaluation of random number packages and observed that Feigenbaum constants successfully pass all these tests. These constants may lend themselves as reliable randomness sources if extended expansions can be obtained.

\section{References}

[1] K. Karamanos, I. Kotsireas, Statistical analysis of the first digits of the binary expansion of Feigenbaum constants $\alpha$ and $\delta$, J. Franklin Inst. 342 (3) (2005) 329-340.

[2] D. Broadhurst, Feigenbaum constants to 1018 decimal places, 22 March 1999, <http://pi.lacim.uqam.ca/ piDATA/feigenbaum.txt $>$

[3] I.J. Good, The serial test for sampling numbers and other tests for randomness, Proc. Cambridge Philos. Soc. 47 (1953) 276-284. 